\title{
Locomotor activity of rats with SCI is improved by dexmedetomidine by targeting the expression of inflammatory factors
}

\author{
WEI-GUO WANG ${ }^{1 *}$, LIN WANG $^{2 *}$, ZHEN-HUA JIAO $^{3}$, BIN XUE $^{4}$ and ZHAN-WANG XU ${ }^{1}$
}

\author{
${ }^{1}$ Department of Orthopedics, The First Affiliated Hospital of Shandong University of Traditional Chinese Medicine, \\ Jinan, Shandong 250014; ${ }^{2}$ Department of Orthopedics, Affiliated Hospital of Taishan Medical University, Taian, Shandong 271016; \\ ${ }^{3}$ Department of Orthopedics, Rizhao Hospital of Traditional Chinese Medicine, Rizhao, Shandong 276800; \\ ${ }^{4}$ Department of Orthopedics, Jinan Hospital of Traditional Chinese Medicine, Jinan, Shandong 250000, P.R. China
}

Received August 10, 2016; Accepted May 15, 2017

DOI: $10.3892 / \mathrm{mmr} .2018 .8930$

\begin{abstract}
Dexmedetomidine, a well-known selective $\alpha-2$ adrenoceptor agonist, inhibits the apoptosis of neurons and protects other organs from oxidative damage. In the present study, the effect of dexmedetomidine on spinal cord injury (SCI) in a rat model was investigated. The SCI rat model was prepared using the weight-drop method, and the effect of dexmedetomidine on locomotor activity was analyzed using the Basso, Beattie and Bresnahan (BBB) rating scale. Western blot analysis was used to observe changes in the expression of apoptosis-related proteins, including B-cell lymphoma 2 (Bcl-2) and Bcl-2-associated X protein (Bax). The results revealed that treatment of the SCI rats with dexmedetomidine at a dose of $50 \mathrm{mg} / \mathrm{kg}$ significantly prevented the formation of edema in the tissues of the spinal cord. Dexmedetomidine also inhibited the SCI-induced accumulation of neutrophils in the spinal cord. The BBB scores were significantly increased $(\mathrm{P}<0.05)$ in the rats with SCI treated with dexmedetomidine after 10 days. The results of grid walking test revealed a marked decrease in the number of missteps following 10 days of dexmedetomidine treatment. The expression levels of tumor necrosis factor (TNF)- $\alpha$ and interleukin (IL)-1 $\beta$ were significantly reduced $(\mathrm{P}<0.05)$ in the spinal cord tissues of the dexmedetomidine group, compared with those in the control group of rats. Dexmedetomidine treatment following SCI exerted an inhibitory effect on the SCI-induced increase in the expression of Bax. The expression of $\mathrm{Bcl}-2$ was increased in
\end{abstract}

Correspondence to: $\mathrm{Dr}$ Zhan-Wang Xu, Department of Orthopedics, The First Affiliated Hospital of Shandong University of Traditional Chinese Medicine, 44 Wenhuaxi Road, Jinan, Shandong 250014, P.R. China

E-mail: xuzhanwang11@hotmail.com

${ }^{*}$ Contributed equally

Key words: locomotor activity, edema, apoptosis, neutrophils, oxidative damage the dexmedetomidine treated rats, compared with that in the control group. Taken together, dexmedetomidine improved the locomotor activity of the rats through the inhibition of edema, reduction in the expression levels of TNF- $\alpha$ and IL-1 $\beta$, and inhibition of the induction of apoptosis. Therefore, dexmedetomidine may be of therapeutic importance for patients with SCI.

\section{Introduction}

Spinal cord injury (SCI) results from the breakdown of spinal cord tissues due to mechanical stress and is a severe health problem worldwide. The primary acute phase of SCI consists of direct tissue lesion and is accompanied by hemorrhage, whereas the secondary phase consists of neuronal apoptosis at the sites of lesion formation $(1,2)$. During the secondary phase of SCI, the generation of reactive oxygen species, formation of edema, expression of cytokines leading to inflammation, and neuronal apoptosis occur (3-5). The onset of inflammation is considered to be the most serious factor resulting in secondary injury (3-5). Neuronal cells do not possess the ability to regenerate following SCI, which requires the treatment strategy be free from any harmful side effects. Therefore, studies have investigated the effect of inhibitors of apoptosis on neurological injuries, including SCI, with the aim of preventing the secondary phase of injury (6).

Cytokines, also known as inflammatory factors, including, tumor necrosis factor (TNF)- $\alpha$ ), interleukin (IL)-6 and IL-18, are vital in various pathological and physiological process in cells. In patients with acute kidney injury, it has been observed that IL-18 acts as a marker for the level of acute kidney injury, and its expression determines patient survival rates (7). The expression of TNF- $\alpha$ and IL- 6 mediated by IL-18 enhance the rate of inflammatory reactions leading to organ injury (8). Therefore, inhibiting the expression of inflammatory factors is important in the prevention of organ injury.

Dexmedetomidine is known for its role as a selective $\alpha-2$ adrenoceptor agonist and it exhibits inhibitory effect on sympathetic activity $(9,10)$. It has been reported that dexmedetomidine inhibits the apoptosis of neurons and protects various other organs from damage $(11,12)$. Dexmedetomidine treatment 
has also been found to prevent liver and intestinal injury in patients following hepatectomy through the inhibition of cell apoptosis $(13,14)$. In the present study, the effect of dexmedetomidine treatment on SCI in a rat model was investigated. It was observed that dexmedetomidine improved locomotor activity in the rats with SCI by inhibiting edema formation, reducing cytokine expression and inhibiting the induction of neuronal apoptosis.

\section{Materials and methods}

Experimental animals. A total of 30 adult male Sprague-Dawley rats (weight, 240-260 g) were obtained from the Experimental Animal Center (Xiamen University, Zhangzhou, China). The animals were housed in well-ventilated rooms with $\sim 60 \%$ humidity, a temperature maintained at $23^{\circ} \mathrm{C}$ and a $12 / 12-\mathrm{h}$ light/dark cycle for 15 days prior to the start of experiments. The experimental procedures were performed in accordance with the Guide for the Care and Use of Laboratory Animals of the National Institute of Health (15). The procedures were approved by the Government Animal Care Committee of the Medical College of Xiamen University under the reference no. 011/MCXU/2013. The animals were sacrificed following anesthetization with an injection of $400 \mathrm{mg} / \mathrm{kg}$ of chloral hydrate (Beyotime Institute of Biotechnology, Haimen, China) intraperitoneally. Following SCI, the animals were placed in their cages with free access to standard food and water.

Preparation of the traumatic SCI animal model using the weight-drop method. The SCI rat model was prepared using the weight-drop method. The rats were divided randomly into three groups of 10 rats: Sham injury, SCI and treatment groups. The rats were anesthetized with an injection of $400 \mathrm{mg} / \mathrm{kg}$ chloral hydrate and the spinal cord of each animal was exposed by T12 spinal laminectomy. Following exposure, an impactor with a diameter of $2.0 \mathrm{~mm}$ was used to perform the weight-drop injury to establish the traumatic SCI animal model. The animals in the sham group were not subjected to the weight-drop step.

Treatment strategy. Following SCI, the animals in the treatment group were injected with a single $50 \mathrm{mg} / \mathrm{kg}$ dose of dexmedetomidine (Sigma-Aldrich; Merck KGaA, Darmstadt, Germany) intraperitoneally immediately after the incision was closed. The animals in the sham and SCI groups received the same volume of normal saline.

Analysis of edema formation in the spinal cord of rats with $S C I$. The accumulation of water (edema formation) in the spinal cord tissues of the rats with SCI was determined by measuring the spinal cord weight. The wet weight of the spinal cord immediately following extraction was recorded. The spinal cord was then dried for $24 \mathrm{~h}$ at $75^{\circ} \mathrm{C}$ and the dry weight was measured. The difference in the wet and dry weight of the spinal cord was used for the determination of edema.

Determination of the activity of myeloperoxidase (MPO). The activity of MPO was analyzed for the determination of neutrophil accumulation in the spinal cord tissues of the rats. Briefly, the spinal cord lysates were treated with the o-dianisidine, $50 \mathrm{mM}$ potassium phosphate buffer and $20 \mathrm{mM} \mathrm{H}_{2} \mathrm{O}_{2}$ mixture.
Absorbance of the solution was recorded spectrophotometrically at $460 \mathrm{~nm}$ to determine the rate of change. The quantity of enzymes $(\mathrm{U} / \mathrm{g})$ consumed to quench the reactive oxygen species was measured.

Behavioral assessments. Improvements in the locomotor activity of rats following dexmedetomidine treatment were analyzed using the method described by Basso et al (16). Three independent observers analyzed the locomotor activities of the rats using the Basso, Beattie and Bresnahan (BBB) locomotor rating scale. The scale is graded into 21 points with the lowest point indicating complete paralysis and the highest point representing normal locomotion. On day 1 post-SCI and every week following SCI, the rats were allowed to walk on an irregularly distributed horizontal wire grid. When walking, the observers carefully recorded the locomotor activity of the rats.

Determination of expression levels of TNF- $\alpha$ and $I L-1 \beta$ in rats. As the rate of inflammatory reactions resulting in organ injury is mediated by the increased expression of TNF- $\alpha$ and IL-6 (8), the present study analyzed the expression levels of TNF- $\alpha$ and IL- 6 . At $24 \mathrm{~h}$ post-SCI, the rats in the treatment groups were weighed, anesthetized, placed in a prone position and then sacrificed via injecting air through the ear vein. A small incision was made in the back of each rat, and the skin was carefully removed, followed by the subcutaneous tissues, to expose the spinal cord. The injured spinal cords were collected, placed into lysis buffer $(5 \mathrm{mg} / 100 \mu \mathrm{l})$ and homogenized on ice. The commercially available TNF- $\alpha$ and IL-1 $\beta$ colorimetric kits (Calbiochem; EMD Millipore, Billerica, MA, USA) were used for determination of the expression levels of TNF- $\alpha$ and IL- $1 \beta$ expression according to the manufacturer's protocol. The determination was performed in triplicate.

Western blot analysis. At $24 \mathrm{~h}$ post-SCI, the rats were weighed, anesthetized, placed in a prone position and then sacrificed via injecting air through the ear vein. A small incision was made carefully in the back of rat, and the skin was removed, followed by subcutaneous tissues, to expose the spinal cord. The injured spinal cord was collected, placed into lysis buffer (5 mg/100 $\mu \mathrm{l}$ ) and homogenized on ice. The homogenized spinal cord tissues were transferred into centrifuge tubes and lysed for $10 \mathrm{~min}$, followed by centrifugation for $20 \mathrm{~min}$ at $4,000 \mathrm{x} \mathrm{g}$ at $4^{\circ} \mathrm{C}$. The supernatants were decanted into the pre-cooled centrifuge tubes. The concentration of proteins was determined by Bicinchoninic Acid protein assay kit (Beijing Solarbio Science \& Technology Co., Ltd., Beijing, China) according to the manufacturer instructions. A total of $2 \mu \mathrm{g}$ protein samples were loaded onto $15 \%$ SDS-polyacrylamide gel and separated by electrophoresis. The separated proteins were then transferred onto nitrocellulose membranes. The membranes were blocked with 5\% non-fat-milk solution and then blotted with appropriate primary antibodies against Bax $(1: 1,000 ; 5023 \mathrm{~S})$ and Bcl-2 (1:1,000; 15071S; both from Cell Signaling Technology, Inc., Danvers, MA, USA). The incubation with primary antibodies was performed overnight at $4^{\circ} \mathrm{C}$ followed by $1 \mathrm{~h}$ incubation with peroxidase-labeled secondary antibody (1:2,000 dilution; 7077S; Cell Signaling Technology, Inc.) at room temperature. The bands were visualized using an enhanced chemiluminescence detection technique (GE Healthcare Life Sciences, 


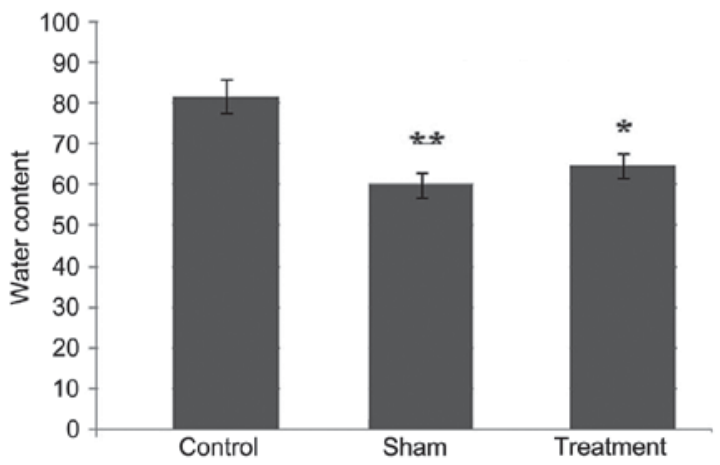

Figure 1. Effect of dexmedetomidine on water content in rats with spinal cord injury. The rats in the treatment group were injected with $50 \mathrm{mg} / \mathrm{kg}$ doses of dexmedetomidine. Analysis of the spinal cord tissues showed a higher content of water in the control group, compared with water content in the sham and treatment groups. ${ }^{*} \mathrm{P}<0.05$ and $^{* *} \mathrm{P}<0.05$, compared with the control group.

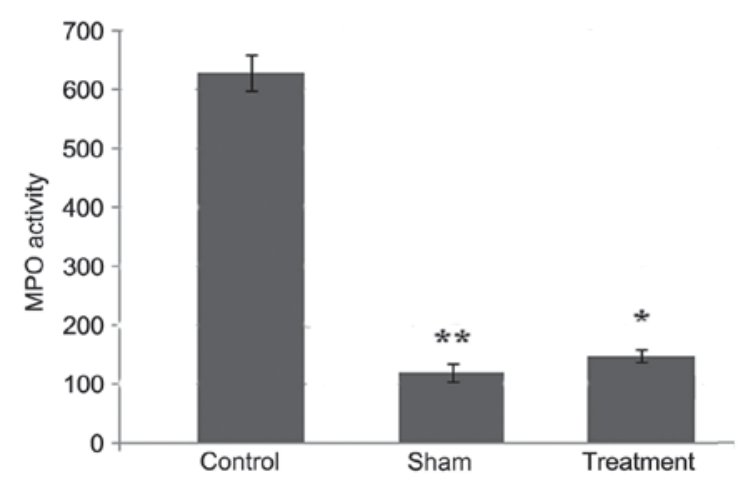

Figure 2. Dexmedetomidine treatment reduces the activity of MPO in the treatment group. The rats following SCI were injected with $50 \mathrm{mg} / \mathrm{kg}$ doses of dexmedetomidine and the spinal cord tissues were analyzed for the activity of MPO. SCI significantly increased the activity of MPO, which was reduced by dexmedetomidine. ${ }^{*} \mathrm{P}<0.05$ and ${ }^{* *} \mathrm{P}<0.05$, compared with the control group. SCI, spinal cord injury; MPO, myeloperoxidase.

Chalfont, UK) using Quantity One Software v4.2 (Bio-Rad Laboratories, Inc., Hercules, CA, USA).

Immunohistochemical assay and $H \& E$ staining. After 10 days post-treatment with dexmedetomidine following SCI, two animals from each group were anesthetized using Nembutal. The thoracic cavities of the rats after opening were intracardially perfused using normal saline. Then, $\sim 400 \mathrm{ml}$ fixative containing paraformaldehyde (4\%) in $0.1 \mathrm{M}$ PBS (pH 7.4) was used for perfusion of the rats and subsequently T6-14 segment was extracted from the animal spinal cord. The spinal cord tissues were treated with phosphate-buffered sucrose $(30 \%)$ following $3 \mathrm{~h}$ of fixing paraformaldehyde (4\%) in $0.1 \mathrm{M}$ PBS at room temperature. The paraffin embedded tissues were mounted on the slides coated with $0.02 \%$ poly-L-lysine. The chromagen used contained a combination of avidin-biotin-peroxidase complex (Invitrogen; Thermo Fisher Scientific, Inc.) and 3,3'-diaminobenzidine hydrochloride (DAB; Sigma-Aldrich; Merck KGaA). For this, the tissues following PBS washing were incubated for $45 \mathrm{~min}$ at room temperature with $1 \%$ bovine serum albumin (Gibco; Thermo Fisher Scientific, Inc.). The tissues were then incubated at $4^{\circ} \mathrm{C}$ for overnight with primary antibodies against platelet-derived growth factor subunit B (PDGF-B; dilution, 1:100; cat. no. 8912; Cell Signaling Technology, Inc.). Following incubation, tissues were washed with PBS and then incubated with biotinylated goat anti-rabbit secondary antibody (diluted to 1:200 in PBS) at room temperature for $1 \mathrm{~h}$. Visualization was performed using DAB $(0.05 \%)$ and hydrogen peroxide $(0.3 \%)$ in combination. Dehydration of tissues using ethanol and xylene was followed by mounting under coverslips. For pathological changes $2 \mu \mathrm{m}$ thick tissue sections were stained with hematoxylin for $5 \mathrm{~min}$ at $25^{\circ} \mathrm{C}$ and with eosin for $2 \mathrm{~min}$ at $25^{\circ} \mathrm{C}$. Stained sections were imaged using a Olympus BX51 light microscope (Olympus Corporation, Tokyo, Japan) (magnification, x400).

Statistical analysis. For statistical analysis, Statistical Package for Social Sciences (SPSS) version 13.0 for Windows (SPSS, Inc., Chicago, IL, USA) was used. One-way analysis of variance was used for the comparison of BBB scores. $\mathrm{P}<0.05$ was considered to indicate a statistically significant difference. Data are expressed as the mean \pm standard error of the mean.

\section{Results}

Dexmedetomidine prevents the formation of edema in rat spinal cord tissues following SCI. The examination of the rats $24 \mathrm{~h}$ following SCI showed the formation of edema due to the accumulation of water content in the spinal cord tissues. However, treatment of the rats with dexmedetomidine at a dose of $50 \mathrm{mg} / \mathrm{kg}$ significantly decreased the formation of edema in the tissues of the spinal cord (Fig. 1). Compared with the rats in the sham and dexmedetomidine treatment groups, edema formation was higher in the spinal cord of rats in the control group.

Dexmedetomidine inhibits the infiltration of neutrophils in the spinal cord tissues. At $24 \mathrm{~h}$ post-SCI, the accumulation of neutrophils was increased, which was evident by a significant increase in the activity of MPO. Treatment of the rats with dexmedetomidine at a dose of $50 \mathrm{mg} / \mathrm{kg}$ exhibited an inhibitory effect on the SCI-induced increase in the accumulation of neutrophils (Fig. 2). Compared with the animals in the control group, the accumulation of neutrophils was significantly $(\mathrm{P}<0.05)$ decreased in the dexmedetomidine and sham groups (Fig. 2).

Effect of dexmedetomidine on functional recovery following $S C I$ in rats. The results from the BBB locomotor test revealed a significant $(\mathrm{P}<0.001)$ increase in locomotor performance 10 days following SCI in the dexmedetomidine treatment group (Fig. 3A). Compared with the first day following SCI, the BBB scores were significantly increased in the rats of the dexmedetomidine group 10 days post-SCI. The comparison of BBB locomotor scores showed the highest score in the sham group, followed by the dexmedetomidine group and then the control group. However, the scores were closer in the animals of the dexmedetomidine and sham groups (Fig. 3A). In the grid walking test, the animals in the control group showed a higher number of missteps, compared with those in the dexmedetomidine treatment group (Fig. 3B). Therefore, a significant 


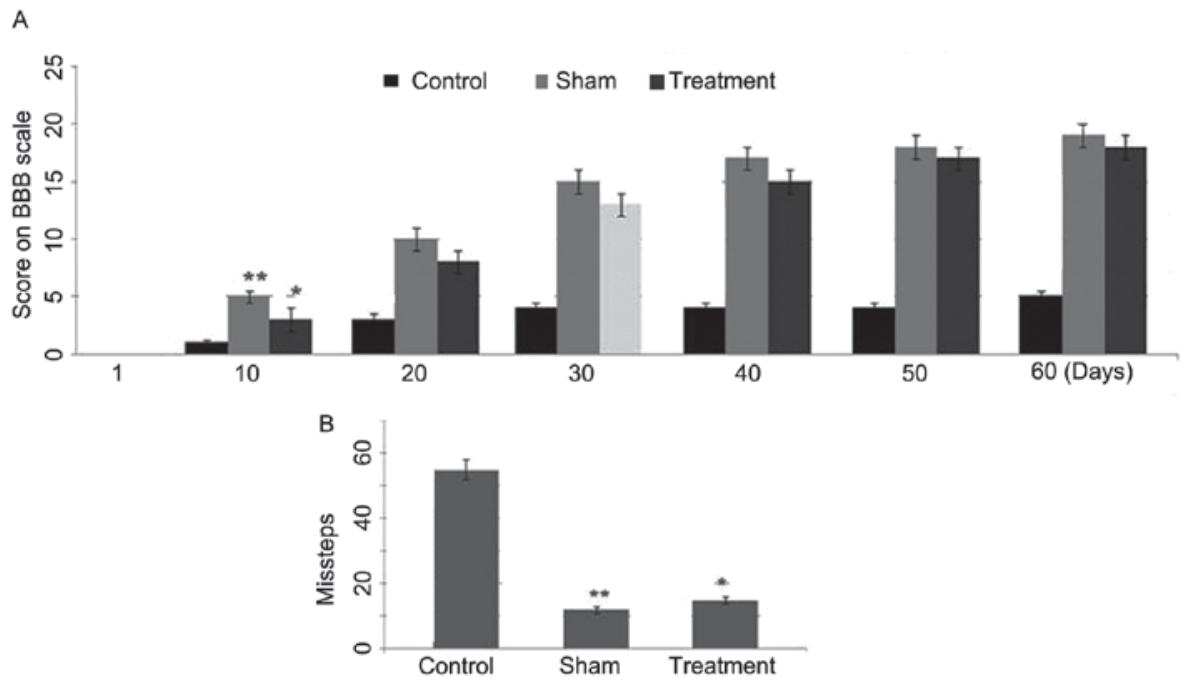

Figure 3. Effect of dexmedetomidine treatment on the improvement of locomotor activity. Locomotor activity was assessed using the (A) BBB score scale and (B) walking wire grid test. Following treatment with dexmedetomidine, rats were analyzed for locomotor activity. The BBB scores were higher in the dexmedetomidine and sham groups, compared with the control group. The number of missteps were significantly higher in the control group, compared with that in the sham and treatment groups. ${ }^{* *} \mathrm{P}<0.01$ and ${ }^{*} \mathrm{P}<0.05$, compared with the control group. BBB, Basso, Beattie and Bresnahan.

A

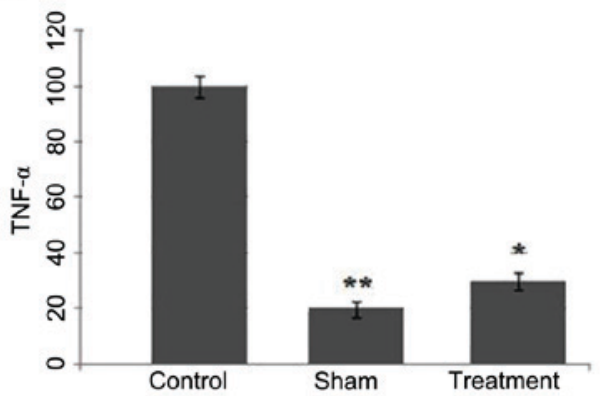

B

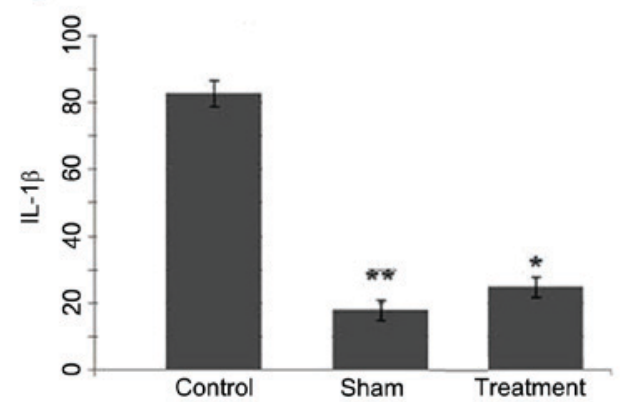

Figure 4. Dexmedetomidine treatment significantly reduces the expression of cytokines in the spinal cord tissues of rats. The rats with spinal cord injury were treated with $50 \mathrm{mg} / \mathrm{kg}$ doses of dexmedetomidine and the spinal cord tissues were analyzed for expression levels of (A) TNF- $\alpha$ and (B) IL-1 $\beta$. TNF- $\alpha$, tumor necrosis factor- $\alpha$; IL-1 $\beta$, interleukin- $1 \beta .{ }^{* *} \mathrm{P}<0.01$ and ${ }^{*} \mathrm{P}<0.05$, compared with the control group.

improvement was observed in the locomotion performance of the animals with SCI treated with dexmedetomidine.

Effect of the dexmedetomidine on the expression levels of TNF- $\alpha$ and IL-1 $\beta$ following SCI. The expression levels of TNF- $\alpha$ and IL-1 $\beta$ in the spinal cord tissues of SCI rats treated with dexmedetomidine were analyzed using western blot analysis. SCI led to a marked increase in the expression of levels of TNF- $\alpha$ and IL- $1 \beta$ at 24 h (control group), compared with the sham group (Fig. 4A and B). However, in the rats treated with dexmedetomidine following SCI, no such increase in the expression levels of TNF- $\alpha$ or IL-1 $\beta$ were observed in the spinal cord tissues (Fig. 4).

Effect of dexmedetomidine on the protein expression levels of $\mathrm{Bax}$ and $\mathrm{Bcl}-2$. Compared with the rats in the sham group, the rats in the control group revealed a marked increase in the expression of Bax following SCI (Fig. 5). Treatment with dexmedetomidine following SCI had an inhibitory effect on the expression of Bax in the tissues of the spinal cord. The expression of Bax in the dexmedetomidine treatment group was similar to that of the control group (Fig. 5). Compared

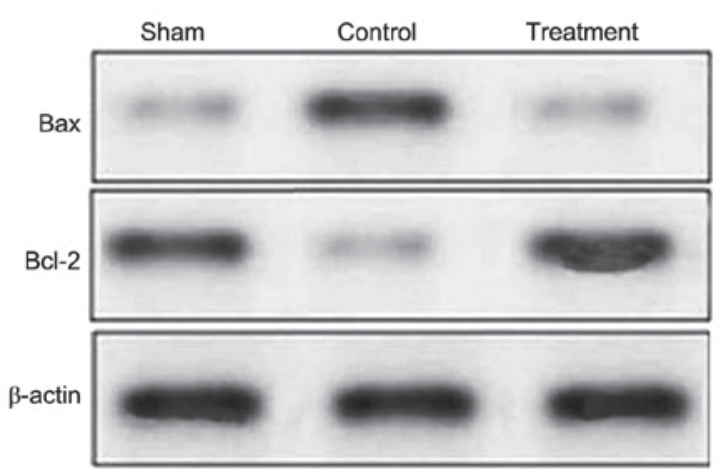

Figure 5. Dexmedetomidine treatment reduces expression of Bax and increases expression of Bcl-2 in the rats with SCI. Treatment with dexmedetomidine following SCI had an inhibitory effect on the expression of Bax in the tissues of the spinal cord. Compared with the sham group, the expression of Bcl-2 was significantly reduced in the rats at $24 \mathrm{~h}$ following SCI. Howeverm the expression of Bcl-2 was significantly increased in the rats with SCI treated with dexmedetomidine. SCI, spinal cord injury.

with the sham group, the expression of Bcl-2 was significantly reduced in the rats $24 \mathrm{~h}$ following SCI, however, the expression 


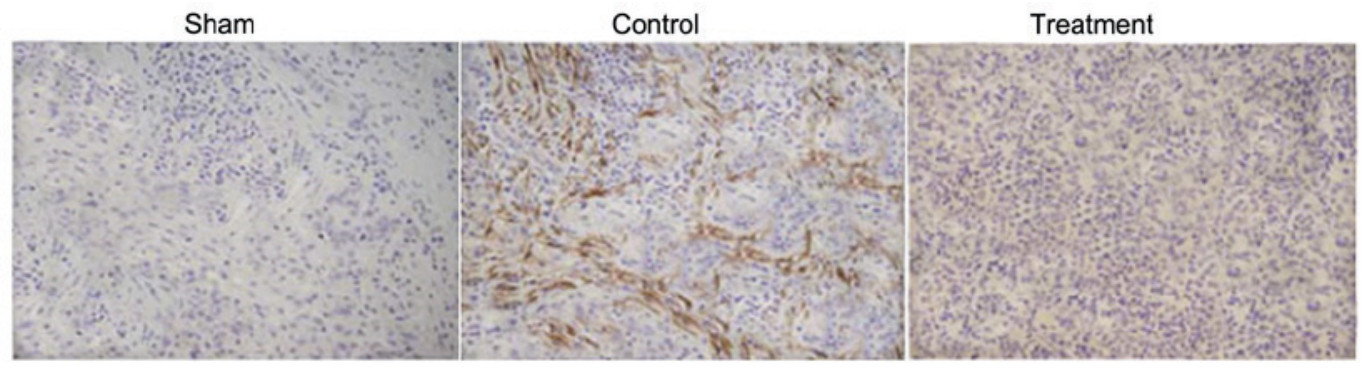

Figure 6. Dexmedetomidine treatment reduces spinal cord injury-induced cell apoptosis. The spinal cord tissues were stained with hematoxylin and eosin and then examined using light photomicrographs (magnification, $\mathrm{x} 400$ ).

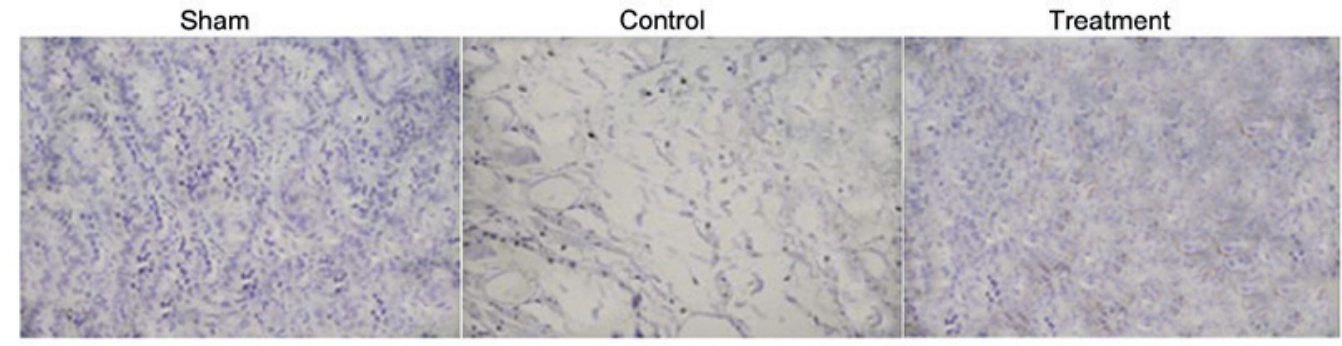

Figure 7. Dexmedetomidine treatment prevents spinal cord injury-induced vacuole formation and tissue degeneration. The spinal cord tissues of the rats were subjected to DAB for PDGF-B immunohistochemical staining (magnification, x400). PDGF-B, platelet-derived growth factor subunit B.

of Bcl-2 was significantly increased in the rats with SCI treated with dexmedetomidine (Fig. 5).

Hematoxylin and eosin staining. The examination of spinal cord tissues using light microscopy revealed the presence of large cavities. The number of neuronal cells undergoing apoptosis and gliocytes increased markedly in the rats with SCI. However, treatment of the rats with dexmedetomidine prevented the formation of cavities, induction of neuronal cell apoptosis and increased number of gliocytes in the injured region (Fig. 6). The results from the immunohistochemical examination showed reductions in the percentage of PDGF-B-positive cells and porosis formation in the spinal cord of rats with SCI. Treatment of the SCI rats with dexmedetomidine increased the migration of PDGF-B-positive cells to the injured region of spinal cord and prevented porosis formation (Fig. 7).

\section{Discussion}

In the present study, the effect of dexmedetomidine on locomotor activity in the rats following SCI was investigated. The study demonstrated a significant improvement in the locomotor activity of rats with SCI treated with dexmedetomidine through the inhibition of edema formation, reduction in neutrophil accumulation, decrease in the expression of cytokines and inhibition of cell apoptosis.

Studies have demonstrated that SCI leads to the development of edema and production of reactive oxygen species, and inhibits the potential of neurons to undergo repair, which is associated with worsening of the disorder (17). These factors are responsible for mediating the onset of secondary processes in cases of SCI. In the present study, dexmedetomidine exhibited an inhibitory effect on the accumulation of neutrophils, which was evident by a significant decrease in the activity of MPO.
Treatment of the rats suffering from SCI with dexmedetomidine led to a significant decrease in the expression of factors

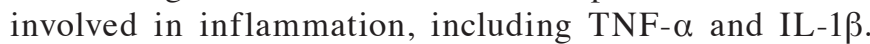
Treatment of mice with SCI with certain chemotherapeutic agents has been shown enhance the regenerative potential of the affected neurons, prevent neuronal apoptosis and induce improvements in motor function $(18,19)$. In the present study, the expression of Bax was reduced, whereas that of Bcl-2 was increased in the spinal cord tissues of SCI rats treated with dexmedetomidine. This suggested that dexmedetomidine inhibited the induction of neuronal apoptosis. Treatment of the SCI rats with dexmedetomidine also led to improvements in the functions of limbs in the rats.

$\mathrm{SCI}$ is followed by the development of inflammation and the subsequent accumulation of neutrophils into the tissues (20). Inflammatory factors are secreted and reactive oxygen species are produced, resulting in neuronal apoptosis and necrosis $(21,22)$. Therefore, the inhibition of cytokine secretion by the spinal cord tissues following SCI can prevent neuronal cell damage. The results from the present study demonstrated a significant decrease in the expression of cytokines TNF- $\alpha$ and IL-1 $\beta$ in the rats with SCI treated with dexmedetomidine.

In conclusion, the results of the present study demonstrated that dexmedetomidine improved the locomotor function of rats with SCI through the inhibition of edema formation, reduction of neutrophil accumulation, inhibited expression of cytokines and inhibited induction of apoptosis. Therefore, dexmedetomidine may be used for the improvement of locomotor function in cases of SCI.

\section{Acknowledgements}

The authors are highly thankful to The First Affiliated Hospital of Shandong University of Traditional Chinese 
Medicine (Jinan, China) for the facilities to complete the research of the present study.

\section{Funding}

No funding was received.

\section{Availability of data and materials}

All data generated or analyzed during this study are included in this published article.

\section{Authors' contributions}

$\mathrm{ZHJ}$ and BX performed the experimental work, whereas ZWX made substantial contributions to the analysis of the obtained data. WGW and LW made substantial contributions to the conception and design of the study and wrote the paper. All authors read and approved the final manuscript.

\section{Ethics approval and consent to participate}

The experimental procedures were performed in accordance with Guide for the Care and Use of Laboratory Animals of the National Institute of Health (15). The procedures were approved by the Government Animal Care Committee of the Medical College of Xiamen University under the reference no. 011/MCXU/2013.

\section{Consent for publication}

Not applicable.

\section{Competing interests}

The authors declare that they have no competing interests.

\section{References}

1. Schmitt C, Miranpuri GS, Dhodda VK, Isaacson J, Vemuganti R and Resnick DK: Changes in spinal cord injury-induced gene expression in rat are strain-dependent. Spine J 6: 113-119, 2006.

2. Hong Z, Chen H, Hong H, Lin L and Wang Z: TSP-1 expression changes in diabetic rats with spinal cord injury. Neurol Res 31: 878-882, 2009.

3. Qiao F, Atkinson C, Kindy MS, Shunmugavel A, Morgan BP, Song $\mathrm{H}$ and Tomlinson $\mathrm{S}$ : The alternative and terminal pathways of complement mediate post-traumatic spinal cord inflammation and injury. Am J Pathol 177: 3061-3070, 2010.

4. Beattie MS: Inflammation and apoptosis: Linked therapeutic targets in spinal cord injury. Trends Mol Med 10: 580-583, 2004

5. Beck KD, Nguyen HX, Galvan MD, Salazar DL, Woodruff TM and Anderson AJ: Quantitative analysis of cellular inflammation after traumatic spinal cord injury: Evidence for a multiphasic inflammatory response in the acute to chronic environment. Brain 133: 433-447, 2010.
6. Grasso G, Sfacteria A, Meli F, Fodale V, Buemi M and Iacopino DG: Neuroprotection by erythropoietin administration after experimental traumatic brain injury. Brain Res 1182: 99-105, 2007.

7. Nisula S, Yang R, Poukkanen M, Vaara ST, Kaukonen KM, Tallgren M, Haapio M, Tenhunen J, Korhonen AM and Pettilä V; FINNAKI Study Group: Predictive value of urine interleukin-18 in the evolution and outcome of acute kidney injury in critically ill adult patients. Br J Anaesth 114: 460-468, 2015.

8. Yang Y, Zhang ZX, Lian D, Haig A, Bhattacharjee RN and Jevnikar AM: IL-37 inhibits IL-18-induced tubular epithelial cell expression of pro-inflammatory cytokines and renal ischemia-reperfusion injury. Kidney Int 87: 396-408, 2015.

9. Miranda ML, Balarini MM and Bouskela E: Dexmedetomidine attenuates the microcirculatory derangements evoked by experimental sepsis. Anesthesiology 122: 619-630, 2015.

10. Geloen A, Chapelier K, Cividjian A, Dantony E, Rabilloud M, May CN and Quintin L: Clonidine and dexmedetomidine increase the pressor response to norepinephrine in experimental sepsis: A pilot study. Crit Care Med 41: e431-e438, 2013.

11. Duan X, Li Y, Zhou C, Huang L and Dong Z: Dexmedetomidine provides neuroprotection: Impact on ketamine-induced neuroapoptosis in the developing rat brain. Acta Anaesthesiol Scand 58: 1121-1126, 2014.

12. Turan A, Bashour CA, You J, Kirkova Y, Kurz A, Sessler DI and Saager L: Dexmedetomidine sedation after cardiac surgery decreases atrial arrhythmias. J Clin Anesth 26: 634-642, 2014

13. Wang ZX, Huang CY, Hua YP, Huang WQ, Deng LH and Liu KX: Dexmedetomidine reduces intestinal and hepatic injury after hepatectomy with inflow occlusion under general anaesthesia: A randomized controlled trial. Br J Anaesth 112: 1055-1064, 2014.

14. Si YN, Bao HG, Xu L, Wang XL, Shen Y, Wang JS and Yang XB: Dexmedetomidine protects against ischemia/reperfusion injury in rat kidney. Eur Rev Med Pharmacol Sci 18: 1843-1851, 2014.

15. Guide for the Care and Use of Laboratory Animals: National Research Council (US) Committee for the Update of the Guide for the Care and Use of Laboratory Animals. 8th edition. National Academies Press, Washington (DC), 2011.

16. Basso DM, Beattie MS and Bresnahan JC: Graded histological and locomotor outcomes after spinal cord contusion using the NYU weight-drop device versus transection. Exp Neurol 139: 244-256, 1996

17. Loane DJ and Byrnes KR: Role of microglia in neurotrauma. Neurotherapeutics 7: 366-377, 2010

18. Yin Y, Sun W, Li Z, Zhang B, Cui H, Deng L, Xie P, Xiang J and Zou J: Effects of combining methylprednisolone with rolipram on functional recovery in adult rats following spinal cord injury. Neurochem Int 62: 903-912, 2013

19. Genovese T, Mazzon E, Crisafulli C, Esposito E, Di Paola R, Muià C, Di Bella $\mathrm{P}$, Meli R, Bramanti $\mathrm{P}$ and Cuzzocrea S: Combination of dexamethasone and etanercept reduces secondary damage in experimental spinal cord trauma. Neuroscience 150: $168-181,2007$.

20. McTigue DM, Tani M, Krivacic K, Chernosky A, Kelner GS, Maciejewski D, Maki R, Ransohoff RM and Stokes BT: Selective chemokine mRNA accumulation in the rat spinal cord after contusion injury. J Neurosci Res 53: 368-376, 1998

21. Zhao W, Xie W, Le W, Beers DR, He Y, Henkel JS, Simpson EP, Yen AA, Xiao Q and Appel SH: Activated microglia initiate motor neuron injury by a nitric oxide and glutamate-mediated mechanism. J Neuropathol Exp Neurol 63: 964-977, 2004.

22. Morino T, Ogata T, Horiuchi H, Takeba J, Okumura H, Miyazaki T and Yamamoto H: Delayed neuronal damage related to microglia proliferation after mild spinal cord compression injury. Neurosci Res 46: 309-318, 2003. 